\title{
Repaired oesophageal atresia: respiratory morbidity and pulmonary function in adults
}

\author{
S. Sistonen*,\#, P. Malmberg ", K. Malmström ${ }^{\star}$, T. Haahtela ", S. Sarna ${ }^{+}$, \\ R.J. Rintala* and M.P. Pakarinen*
}

ABSTRACT: Although after oesophageal atresia (OA) repair in infancy, respiratory problems are common, their natural history remains unclear. We assessed morbidity, pulmonary function (PF), and bronchial hyperresponsiveness (BHR) in adults with repaired OA respiratory.

588 patients who underwent surgery for OA during 1947-1985 were identified and those 262 who were alive and had their native oesophagus were included. Respiratory symptoms and respiratory symptom-related quality of life (RSRQoL) were assessed by questionnaire and interview, and the patients underwent spirometry, a histamine challenge test, and an exhaled nitric oxide test. For the questionnaires, we added 287 carefully matched general populationderived controls.

Among the 101 (58 male) patients, median age 36 yrs (range 22-56 yrs), respiratory morbidity was significantly increased compared to controls. Patients had more respiratory symptoms and infections, as well as asthma and allergies, and more often impaired RSRQoL ( $p<0.001$ for all). PF tests revealed restrictive ventilatory defect in $21(21 \%)$ patients, obstructive ventilatroy defect in $21(21 \%)$ patients, and both in $36(36 \%)$ patients. A total of $41(41 \%)$ had BHR, and in $15(15 \%)$, it was consistent with asthma. The most significant risk factors for restrictive ventilatory defect were thoracotomy-induced rib fusions (OR 3.4, 95\% $\mathrm{Cl} 1.3-8.7 ; p=0.01)$ and oesophageal epithelial metaplasia (OR 3.0, 95\% Cl 1.0-8.9; $p=0.05$ ).

After repair of $O A$, respiratory-related morbidity, restrictive ventilatory defect and BHR extended into adulthood. Nearly half the patients had BHR and over half had a restrictive ventilatory defect. Thoracotomy-induced rib fusions and gastro-oesophageal reflux-associated oesophageal epithelial metaplasia were the strongest risk factors for restrictive ventilatory defect.

KEYWORDS: Atopy, bronchial hyperresponsiveness, long-term outcome, oesophageal atresia, pulmonary function, tracheo-oesophageal fistula

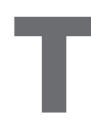
he most common congenital anomaly of the oesophagus is oesophageal atresia (OA). It is usually associated with a tracheo-oesophageal fistula (TOF) [1, 2] and affects approximately one in 2,500 live births [3]. The embryology of OA is incompletely understood [4], and over half these infants have associated anomalies [3,5]. The malformation itself and surgical trauma result in impaired oesophageal motility, a dysmotility predisposing to gastro-oesophageal reflux (GOR) and its complications [6, 7]. A strong connection exists between severity of GOR and persistence of respiratory symptoms among OA survivors [4], but respiratory problems become less frequent with time.

After repair of OA, approximately one-third of these patients suffer respiratory symptoms in adolescence and adulthood [8-12]. Prevalence of wheeze ranges $26-43 \%[10,13,14]$, and that of doctor-diagnosed asthma (DDA) 12-29\% [9, 10, 12, $14,15]$. This is generally higher than in children $(8.8 \%)$ [16] and adults (6\%) [17]. Additionally, pulmonary function (PF) abnormalities, mostly mild restrictive disease [14, 18, 19], occur after repair of OA in childhood [14], adolescence [9, 18, $20,21]$ and adulthood $[12,19,21]$. Restriction may result from surgical trauma, repeated aspiration or recurrent chest infections, as well as from associated thoracic musculoskeletal defects, such as postoperative rib fusions, scoliosis or other chest deformities.

Although after repair of OA, respiratory symptoms are common, the long-term outcome of PF and respiratory morbidity remain unclear. As growing numbers of OA survivors are reaching adulthood, interest in their long-term outcome is increasing. To this end, we performed a population-based, long-term follow-up study in
AFFILIATIONS

${ }^{*}$ Section of Paediatric Surgery,

Hospital for Children and

Adolescents,

'Dept of Allergy, Helsinki University Central Hospital,

${ }^{+}$Dept of Public Health, University of

Helsinki, Helsinki, and

\#Dept of Surgery, Päijät-Häme

Central Hospital, Lahti, Finland.

CORRESPONDENCE

S. Sistonen

Section of Paediatric Surgery,

Hospital for Children and

Adolescents

Helsinki University Central Hospital

P.0. Box 281

FI-00029-HUS

Finland

E-mail: saara.sistonen@helsinki.fi.

Received:

Sept 282009

Accepted after revision:

Feb 212010

First published online:

March 292010 
adults with repaired OA, assessing respiratory morbidity, respiratory symptom-related quality of life (RSRQoL), PF, bronchial responsiveness and atopy. The predictive factors for restrictive ventilation in adulthood were also studied.

\section{PATIENTS AND METHODS}

The hospital records of all 588 patients treated for OA at the Hospital for Children and Adolescents, Helsinki University Central Hospital (Helsinki, Finland) between 1947-1985 were reviewed. Vital status and postal addresses came from the database of the Population Register Centre of Finland based on a personal identification code given to all residents of Finland. Of the 588 infants who underwent surgery for OA, 296 were alive at the beginning of the study in November 2005. The 34 who had undergone oesophageal replacement were excluded. 16 patients had emigrated, and the postal addresses of 11 were unknown. The remaining 235 (90\%) eligible survivors with a native oesophagus were contacted by mail. Letters described the study protocol and purpose. Those returning signed consent forms received invitations to enter the study. Data drawn from the hospital records included survival, type of OA, associated anomalies, surgical treatment and complications.

During their outpatient visit, each participant filled in a symptom questionnaire and underwent an interview, clinical assessment, flow-volume spirometry for PF, histamine challenge test (HCT) for bronchial responsiveness, exhaled nitric oxide fraction $(\mathrm{FeNO})$ for airway inflammation, skin prick test (SPT) for common allergens, and measurement of serum level of immunoglobulin (Ig)E.

The present study is part of a larger evaluation of the longterm outcomes of OA. Findings concerning oesophageal morbidity and function [22], incidence of cancer [23], and natural history of spinal anomalies and scoliosis [24] among adults with repaired OA have already been published. GOR symptoms occurred in 34 of the study participants, and 85 had symptoms of dysphagia due to oesophageal dysmotility after repair of OA; these occurrences were significantly more frequent than among the general population-derived controls [22]. A total of 21 adults with repaired OA had oesophageal epithelial metaplasia in their oesophageal biopsy samples [22]. In addition, chest wall deformities were noted in 15 study participants [24].

\section{Interview and questionnaires}

All participants were interviewed by the same investigator with a structured form, providing information on medical history, respiratory symptoms and diseases, symptoms of allergy, need for asthma medication, and smoking habits. Symptoms of respiration included wheeze, attacks of shortness of breath, recurrent cough and respiratory infections. Symptoms of allergy included allergic rhinitis, conjunctivitis, rash and atopic eczema. The questionnaire was a modification of validated questionnaires on asthma and allergy symptoms [25] as well as on RSRQoL [26]. The identical symptom questionnaire was sent to a group of 915 controls randomly chosen from the Population Register Centre of Finland, matched for age, sex and municipality of residence.

\section{Pulmonary function, histamine provocation and exhaled nitric oxide}

Flow-volume spirometry was performed according to the guidelines of the European Respiratory Society (ERS) [27], and bronchial hyperresponsiveness (BHR) was estimated using a validated dosimetric HCT [28]. Forced vital capacity (FVC) and forced expiratory volume in $1 \mathrm{~s}$ (FEV1) were recorded, as well as their reference value percentages. We used national reference values for spirometry published previously [29]. Ventilatory function was defined as restrictive when FVC was $<80 \%$, which corresponds to a z-score of -2.0 , and obstructive when FEV1/FVC was $<87 \%$ predicted, which also corresponds to a z-score of -2.0. Defects were classified as mild (z-score $-2.0-3.5$ ), moderate (z-score -3.5- -5.5), or severe (z-score -5.5- -7.5). Through use of the dose-response curve, the provocative dose of inhaled histamine producing a decrease of $15 \%$ in FEV1 (PD15) could be determined, and, based on bronchial hyperreactivity, was graded as mild (0.41-1.60 mg), moderate $(0.11-0.40 \mathrm{mg})$ or severe $(\leqslant 0.1 \mathrm{mg})$ [30]. Patients with PD15 $\leqslant 1.6 \mathrm{mg}$ were considered to have increased bronchial responsiveness. Previous study has shown that moderate to severe BHR (PD15 $\leqslant 0.40 \mathrm{mg}$ ) has a high specificity for asthma [28].

FeNO was measured using computerised equipment with a chemiluminescence analyser (Niox; Aerocrine AB, Sweden), according to American Thoracic Society (ATS) recommendations [30]. The patients were seated, wore no nose clip, and were asked to fill their lungs completely with NO-free air, and thereafter to exhale with a mean $\pm 10 \%$ and instantaneous flow of $50 \pm 5 \mathrm{~mL} \cdot \mathrm{s}^{-1}$. An exhalation time of $10 \mathrm{~s}$ served as the default. The system was calibrated with a certified NO calibration gas mixture according to manufacturer's instructions. Exhalations not meeting ATS requirements were rejected by the system, and the patient was asked to perform new exhalation manoeuvres, until providing three reproducible FeNO values within $10 \%$ of each other. The mean FeNO of three acceptable end-expiratory plateau measurements was calculated and recorded [31, 32]. Previously specified reference values for adult lifelong never-smokers, according to the distribution of FeNO by similar technique, and exhalation rate depending on age and height, were used [33].

\section{Atopy}

Atopy was defined by a positive SPT to common allergens. To evaluate sensitisation to common allergens, SPTs were carried out for the following common allergens: birch, grass, mugwort, cat, dog, cow, horse, Cladosporium herbarum, and Dermatophagoides pteronyssinus (Soluprick SQ; ALK, Horsholm, Denmark). The patient was regarded as atopic if one or more reactions were positive, determined as a wheal diameter of $\geqslant 3 \mathrm{~mm}$ in the presence of an expected response to control solutions; the positive control was histamine dihydrochloride $\left(10 \mathrm{mg} \cdot \mathrm{mL}^{-1}\right)$ and as the negative control was saline. The level of serum $\operatorname{IgE}$ was determined based on the standard values of our laboratory, $0-110 \mathrm{kU} \cdot \mathrm{L}^{-1}$ for adults.

\section{Statistical analysis}

Data was analysed using SPSS 16.0 (SPSS Inc., Chicago, IL, USA). Statistical analysis used the Chi-squared and KruskalWallis tests. Data are given as frequencies or as mean and 
range. The multivariate logistic regression model for occurrence of restrictive ventilation defect, included age, any other associated anomaly, rethoracotomies, thoracotomy-induced rib fusions, oesophageal anastomotic leakage, recurrent TOF, primary pulmonary complications, oesophageal epithelial metaplasia and smoking. A study of the urban adult population of Helsinki served as a comparison group for SPTs [34]. Level of significance was set at $\mathrm{p}<0.05$.

\section{Ethics}

The ethics committee of the Hospital for Children and Adolescents, Helsinki University Central Hospital approved this study. Written informed consent was obtained from each participant.

\section{RESULTS}

\section{Subjects}

A total of $72 \%$ of the 235 subjects contacted replied, and the first $101(43 \%)$ patients who returned the signed informed consent form were invited to enter the study (fig. 1). Most of those participating (91\%) had OA with distal TOF. Because patients who had undergone oesophageal replacement were excluded, each patient had a native oesophagus. Of the 101 participating patients, 95 had undergone primary oesophageal anastomosis, two had undergone a staged anastomosis, and

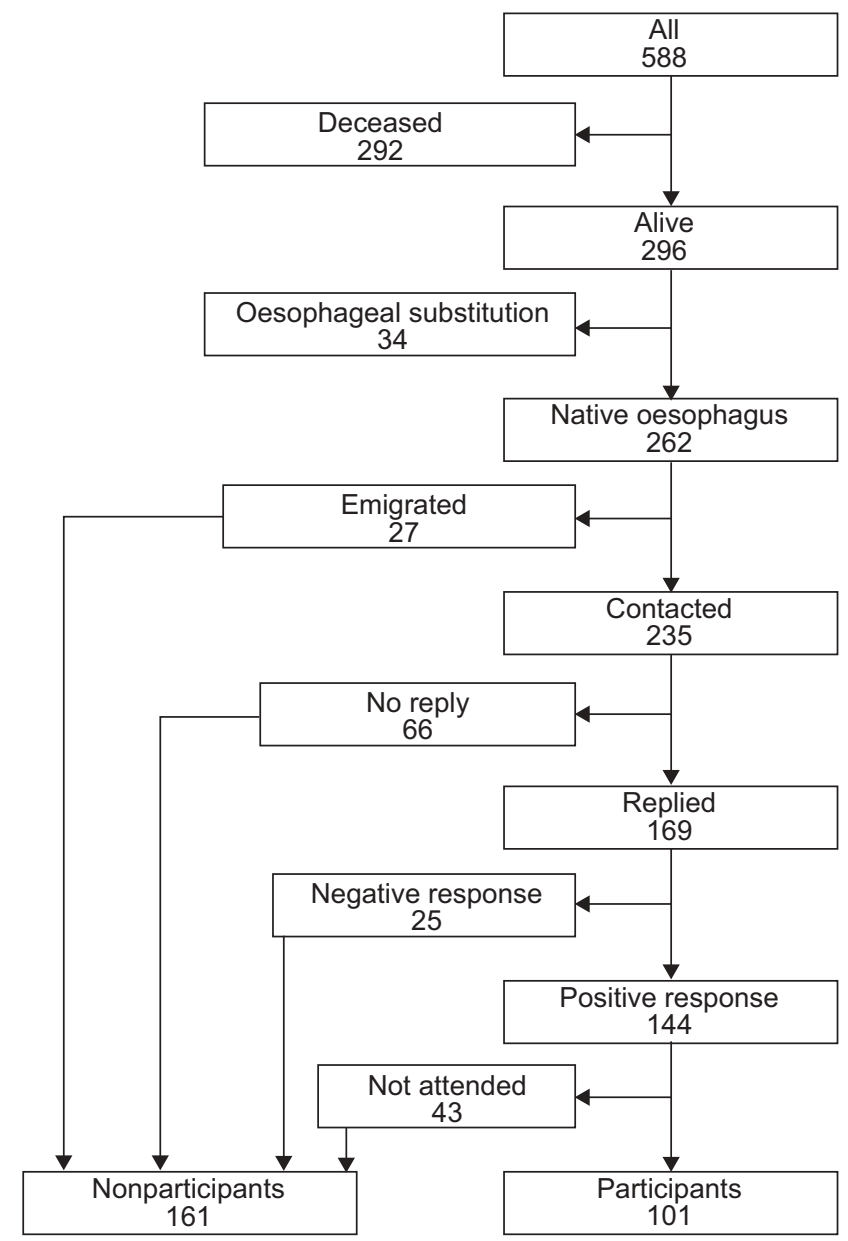

FIGURE 1. Flow chart of study design and patient characteristics. three had undergone a closure of an isolated H-type fistula. Myotomy of the proximal oesophageal pouch to enable primary anastomosis was employed in five subjects. Of the 101 participants, 58 were male, and the mean follow-up time was 36 yrs (range 21-57 yrs) (table 1). Participants and nonparticipants were comparable in terms of age, sex, type of OA, associated anomalies and surgical complications ( $\mathrm{p}>0.05)$. A total of $287(31 \%)$ of the controls responded to the mail questionnaires (table 1).

\section{Tracheopulmonary morbidity in infancy}

Of the patients, 34 had suffered pneumonia as a primary pulmonary complication and 32 needed no postoperative ventilation, with the mean postoperative ventilation time being 2.6 days (range 0-7 days) (table 2). During childhood, tracheomalacia was diagnosed in 15 of the patients, but only one had undergone aortopexy. A total of 10 patients had recurrent TOF.

\section{Questionnaires}

Participants reported increased incidence of allergy, pneumonia, bronchitis, repeated childhood infections, persistent cough and DDA in relation to controls ( $p<0.001$ for all). Among the patients, RSRQoL was significantly decreased $(p<0.001)$. Occurrence of tracheomalacia in infancy was associated with neither current respiratory symptoms nor with tendency to respiratory infections $(\mathrm{p}>0.05)$ (table 3$)$.

\section{Pulmonary function, histamine provocation and exhaled nitric oxide}

In flow-volume spirometry, 21 patients had restriction only, 21 had only obstruction only and 36 had both. Accordingly, 57\% of the patients had restrictive ventilatory defect. The PF test (PFT) results were similar in males and females $(\mathrm{p}>0.05)$ (table 4). A total of 41 had BHR. BHR was mild in 26 patients, moderate in 11 and severe in four; 15 patients had moderate to severe BHR compatible with asthma. BHR was associated with atopy $(\mathrm{p}<0.007)$ and with current respiratory symptoms $(\mathrm{p}<0.03)$. FeNO was clearly elevated only in four patients, and slightly elevated in seven of the patients, but was associated neither with BHR, atopy, current respiratory symptoms, nor PF abnormalities.

\section{Atopy}

37 patients had at least one positive reaction to common allergens in SPT, and sensitisation to multiple allergens was evident in 22. Positive SPT reactions to the most common allergens were $\operatorname{dog}(28 \%)$, cat $(22 \%)$, birch $(23 \%)$, mugwort $(22 \%)$, and timothy grass $(21 \%)$. Elevated levels of serum IgE $>110 \mathrm{kU} \cdot \mathrm{L}^{-1}$ occurred in 20 patients (median $285 \mathrm{kU} \cdot \mathrm{L}^{-1}$, range $126-2,436 \mathrm{kU} \cdot \mathrm{L}^{-1}$ ). Elevated $\operatorname{IgE}$ was associated with multiple sensitisations $(\mathrm{p}<0.001)$, current respiratory symptoms $(p=0.015)$ and BHR $(p<0.007)$, whereas multiple sensitisations were associated with current respiratory symptoms $(p=0.008)$.

\section{Predictors of restrictive ventilation}

Restrictive ventilation defect was only associated with the occurrence of oesophageal epithelial metaplasia in adulthood $(p=0.038)$, but not with tracheomalacia in infancy, history of primary pneumonia, age, airway inflammation in adulthood, or current respiratory symptoms ( $p>0.05$ for each). In a multivariate logistic regression analysis, thoracotomy-induced 
TABLE 1 Characteristics of the participants and nonparticipants with repaired oesophageal atresia $(\mathrm{OA})$ and tracheooesophageal fistula (TOF), and general population-derived controls

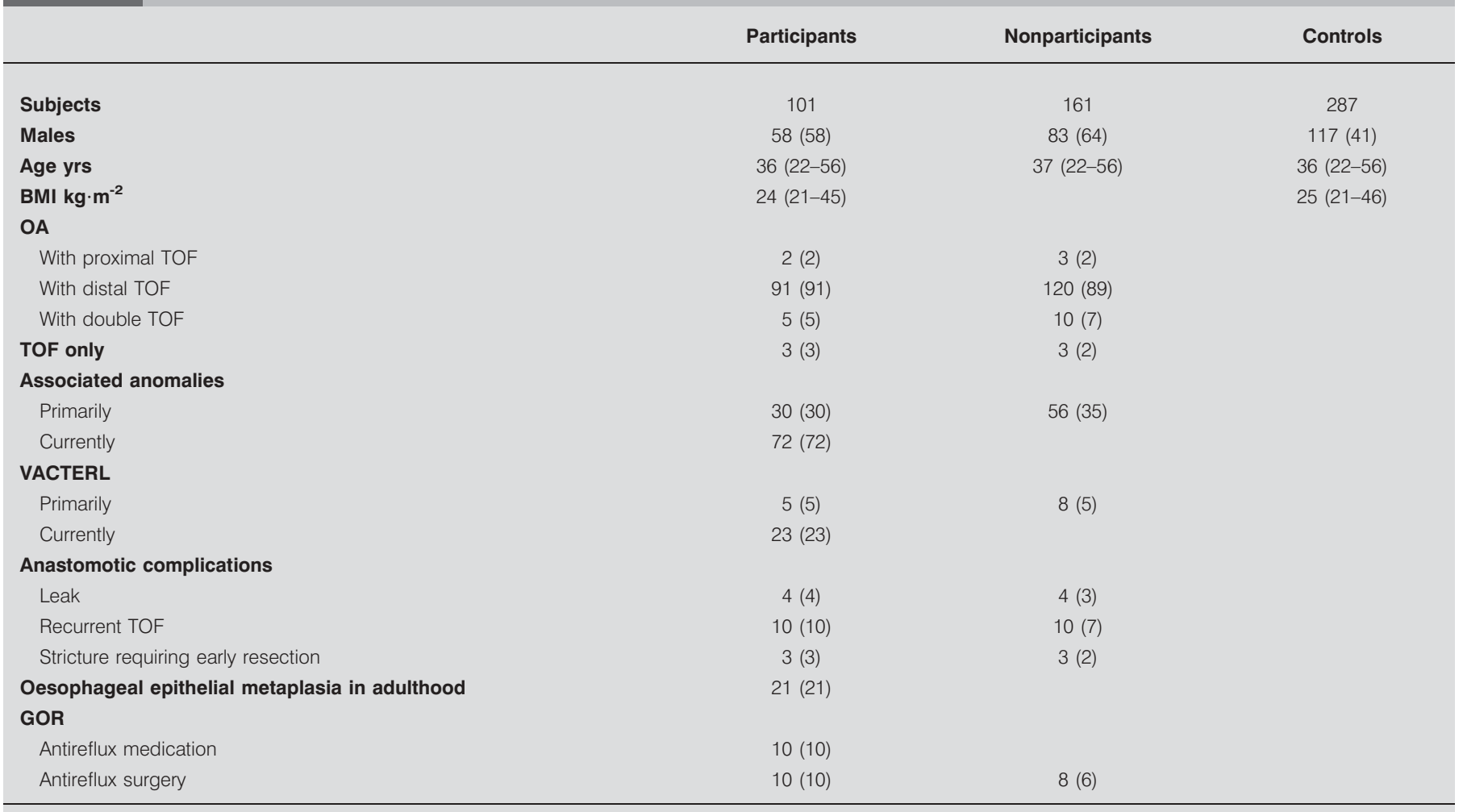

Data are presented as $n, n$ (\%) or mean (range). BMI: body mass index; VACTERL: vertebral defects, anal atresia, cardiovascular anomalies, TOF with OA, renal dysplasia and limb anomalies; GOR: gastro-oesophageal reflux. No significant differences between the groups (Chi-squared test $\mathrm{p}>0.1$ ).

rib fusions (OR 3.4, 95\% CI 1.3-8.7; $\mathrm{p}=0.01$ ) and GORassociated oesophageal epithelial metaplasia in adulthood (OR 3.0, 95\% CI 1.0-8.9; $\mathrm{p}=0.05$ ) were the most significant risk factors for restrictive ventilation defect. Neither increasing

\begin{tabular}{lcc}
\hline TABLE 2 & $\begin{array}{l}\text { Tracheo-pulmonary } \\
\text { oesophageal atresia among participants and } \\
\text { nonparticipants }\end{array}$ \\
& Participants & Nonparticipants \\
\hline Subjects & 101 & 161 \\
Primary pneumonia & $34(34)$ & $47(33)$ \\
Need for postoperative ventilation & & \\
$\quad$ None & $33(33)$ & $66(41)$ \\
1 day & $6(6)$ & $25(16)$ \\
2 days & $22(22)$ & $27(17)$ \\
$3-6$ days & $26(26)$ & $31(19)$ \\
$>7$ days & $13(13)$ & $15(9)$ \\
$\quad$ Tracheostomy & $1(1)$ & 0 \\
Tracheomalacia & $15(15)$ & $13(8)$ \\
Aortopexy & $1(1)$ & $4(2)$ \\
Recurrent TOF & $10(10)$ & $10(7)$ \\
\hline
\end{tabular}

Data are presented as $n$ or $n(\%)$. TOF: tracheo-oesophageal fistula. No significant differences (Chi-squared test $p>0.076$ ). age, sex, other associated anomalies, primary lung complications, primary surgical complications, nor number of thoracotomies, predicted the incidence of restriction.

\section{DISCUSSION}

To the best of our knowledge, this is the largest and only population-based study on respiratory morbidity and $\mathrm{PF}$ in adults with repaired OA. We used validated questionnaires with controls derived from the general population that were carefully matched for age, sex, and municipalities of residency. A drop-out analysis between participants and nonparticipants suggested that the two groups were comparable with respect to primary disease characteristics, minimising the risk of selection bias. We found an increased incidence of daily respiratory symptoms and asthma, as well as decreased RSRQoL. PF abnormalities were detectable in $78 \%$ of the patients: restriction in $57 \%$, obstruction in $55 \%$ and both these ventilation defects in $36 \%$. Occurrence of restrictive PF after repair of OA was associated with thoracotomy-induced rib fusion, which was present in one-third, and GOR-associated oesophageal epithelial metaplasia in one-fifth of the patients. However, the restrictive ventilation defect showed no association with current respiratory symptoms.

Environmental factors, such as cold climate, and inhaled allergens and irritants, have a substantial effect on respiratory symptoms among the general population [35]. In Finland, prevalence of DDA among children is $8.8 \%$ [16] and is $6 \%$ 


\begin{tabular}{|c|c|c|c|c|}
\hline \multirow[t]{2}{*}{ TABLE 3} & \multicolumn{4}{|c|}{$\begin{array}{l}\text { Self-reported occurrence of asthma, allergy and } \\
\text { symptoms of the adult patients with repaired } \\
\text { oesophageal atresia }(n=101) \text { who participated } \\
\text { the clinical studies, and of controls }(n=287)\end{array}$} \\
\hline & & Patients & Controls & $p$-value \\
\hline \multicolumn{2}{|c|}{ Impaired RSRQoL } & 11 & 6 & 0.001 \\
\hline \multicolumn{2}{|c|}{$\begin{array}{l}\text { Current respiratory } \\
\text { symptoms }\end{array}$} & 11 & 2 & 0.001 \\
\hline \multicolumn{2}{|l|}{ DDA } & 16 & 6 & 0.001 \\
\hline \multicolumn{2}{|l|}{ Wheeze } & 37 & 30 & NS \\
\hline \multicolumn{2}{|l|}{ Allergy } & 42 & 11 & 0.002 \\
\hline \multicolumn{2}{|c|}{ Persistent cough } & 31 & 8 & 0.001 \\
\hline \multicolumn{2}{|c|}{ Pneumonia } & 56 & 20 & 0.001 \\
\hline \multicolumn{2}{|l|}{ Bronchitis } & 70 & 50 & 0.001 \\
\hline \multicolumn{2}{|c|}{$\begin{array}{l}\text { Recurrent respiratory } \\
\text { infections }\end{array}$} & 52 & 23 & 0.001 \\
\hline \multicolumn{2}{|c|}{$\begin{array}{l}\text { Childhood respiratory } \\
\text { infections }\end{array}$} & 35 & 13 & 0.001 \\
\hline
\end{tabular}

Data are presented as \%, unless otherwise stated. RSRQoL: respiratory symptom-related quality of life index, as defined in [27]; DDA: doctordiagnosed asthma.

among adults [17], whereas in patients with OA it is even higher $[9,10,12,14,15]$. In the present study, $16 \%$ of the adult patients with repaired OA and $6 \%$ of the general populationderived controls have DDA. These figures are in line with other findings $[9,10,12,14,15]$. Self-reported wheeze occurred in $37 \%$ of the patients and in $30 \%$ of the controls. Self-reported wheeze usually occurs in $\sim 37 \%$ of the survivors of OA with no tendency toward improvement with age $[4,10,11,13,14]$.

In the present study, occurrence of respiratory symptoms was clearly lower among the adult study population than in children and adolescents with repaired OA [9, 10, 12], suggesting that prevalence of respiratory problems decreases with age. Nevertheless, decreased RSRQoL and daily respiratory symptoms were still significantly more common among patients than among controls. Incidences of asthma and BHR were in line with previous results, whereas the PF abnormalities were more common among our adults than in children and adolescents $[9,14,18,20,21,36]$, in accordance with other findings among adults [19].

The proportion of children and adolescents with repaired OA having a restrictive PF defect has ranged $21-40 \%$, and $16-54 \%$ with obstructive ventilation defect $[9,18,20,21,36]$. Our respective figures were $57 \%$ and $55 \%$. PF abnormalities have not shown a correlation with respiratory or oesophageal symptoms $[9,21,36]$. Although a clear link exists between severity of GOR and persistence of respiratory symptoms among OA survivors [4], the reason for PF abnormalities is unclear. They have been suggested to result from recurrent aspiration [21], from poor tracheal clearance leading to recurrent episodes of bronchitis and pneumonia causing lung damage $[15,36]$, or from poor lung growth during infancy [18]. In the present study, thoracotomyinduced rib fusion and GOR-associated epithelial metaplasia of the oesophagus were significant predictors of restrictive ventilation defect. Thus, epithelial metaplasia served as a surrogate marker for significant GOR, which may result in repeated aspiration and lung damage. However, rib fusion leads to an immobile and thus restrictive thoracic cage.

\section{TABLE 4}

Pulmonary function, bronchial hyperresponsiveness, exhaled nitric oxide, and skin prick test (SPT) among adult patients with repaired oesophageal atresia $(n=101)$

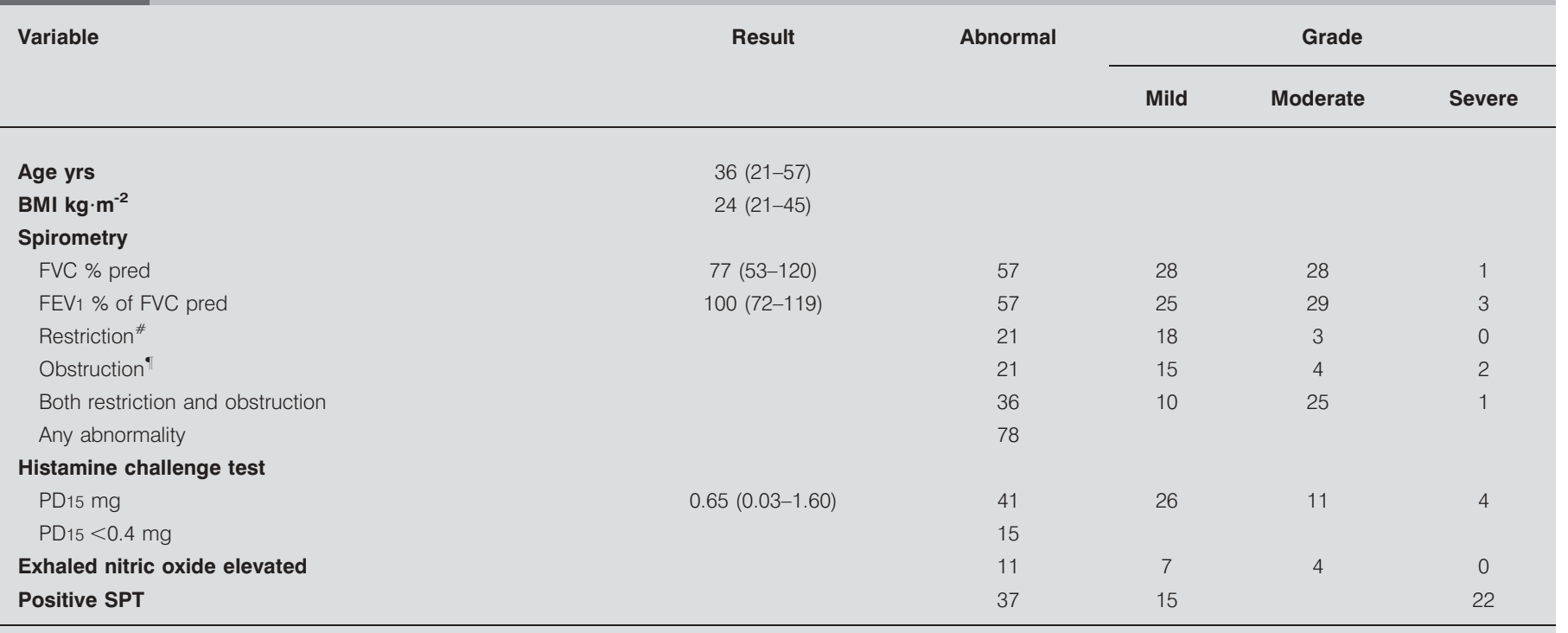

Data are presented as mean (range) or \%. BMI: body mass index; FVC: forced vital capacity; \% pred: \% predicted; FEV1: forced expiratory volume in $1 \mathrm{~s}$ : PD15: provocative dose of histamine causing a $15 \%$ fall in FEV1. ${ }^{\#}$ : restriction is defined as FVC $<80 \%, z$-score $<-2.0$; ${ }^{\circ}$ : obstruction is defined as FEV $1 / F_{V C}<87 \%$, z-score $<-2.0$ 
Prevalence of BHR in the general Finnish population with no previous diagnosis of asthma or chronic bronchitis and with normal lung volumes is $17 \%$ [37]. In our study population, BHR occurred markedly more frequently in $41 \%$, which is in accordance with the results of other studies $[9,18,20,36]$. It has been postulated that increased bronchial reactivity in these patients would merely reflect sequalae of chronic lung disease from damaged epithelium in the airways caused by recurrent aspiration of acidic gastric contents [18]. Severe or moderate BHR has been associated with a more restrictive ventilatory defect among adolescents [9], but no correlation has emerged between increased BHR and history of asthma or atopic eczema $[9,36]$. In the present study, BHR was associated with current respiratory symptoms and atopy. Atopy prevalence was $37 \%$, and $22 \%$ had multiple sensitisations, in accordance with incidences of $34-47 \%$ and $16-42 \%$ among the general population, respectively [34].

Increased $\mathrm{FeNO}$ is associated with the steroid-responsive eosinophilic airway inflammation, higher particularly in patients with atopic asthma [32]. Elevated FeNO was detectable in $23 \%$ of the adolescents with repaired OA [9]. Elevated values were detectable only in patients with atopy and increased BR, but with no relation to respiratory symptoms [9]. Among the present study population, elevated FeNO occurred in $11 \%$ of subjects. FeNO was associated with neither current respiratory symptoms, atopy, nor pulmonary abnormalities. In short, in adult patients with repaired OA, airway inflammation was uncommon in the absence of atopy.

In conclusion, respiratory-related morbidity, restrictive ventilatory defect and BHR extend into adulthood after repair of $\mathrm{OA}$ in a significant number of patients, whereas obstructive ventilatory defect is not the main pulmonary problem. Nearly half the patients had BHR and over half had restrictive ventilatory defect. Thoracotomy-induced postoperative rib fusions and GOR-associated oesophageal epithelial metaplasia in adulthood were the strongest risk factors for restrictive ventilatory defect.

\section{SUPPORT STATEMENT}

This study was supported by the Foundation for Paediatric Research, Foundation for Gastroenterologic Research, Helsinki University Central Hospital, and Central Hospital of Päijät-Häme (all Finland).

\section{STATEMENT OF INTEREST}

None declared.

\section{REFERENCES}

1 Haight C, Towsley HA. Congenital atresia of esophagus with tracheoesophageal fistula. Extrapleural ligation and end-to-end anastomosis of esophageal segments. Surg Gynecol Obstet 1943; 76: 672-688.

2 Waterston DJ, Carter RE, Aberdeen E. Oesophageal atresia: tracheo-oesophageal fistula. A study of survival in 218 infants. Lancet 1962; 21: 819-822.

3 Spitz L. Oesophageal atresia. Orphanet J Rare Dis 2007; 11: 24-36.

4 Goyal A, Jones MO, Couriel JM, et al. Esophageal atresia and tracheo-esophageal fistula. Arch Dis Child Fetal Neonatal Ed 2006; 91: 381-384

5 Chittmittrapap S, Spitz L, Kiely EM, et al. Esophageal atresia and associated anomalies. Arch Dis Child 1989; 64: 364-368.
6 Spitz L, McLeod E. Gastroesophageal reflux. Semin Pediatr Surg 2003; 12: 237-240.

7 Rintala RJ, Sistonen S, Pakarinen MP. Outcome of oesophageal atresia beyond childhood. Semin Pediatr Surg 2009; 18: 50-56.

8 Kovesi T, Rubin S. Long-term complications of congenital esophageal atresia and/or tracheoesophageal fistula. Chest 2004; 126: 915-925.

9 Malmström K, Lohi J, Lindahl H, et al. Longitudinal follow-up of bronchial inflammation, respiratory symptoms, and pulmonary function in adolescents after repair of esophageal atresia with tracheoesophageal fistula. J Pediatr 2008; 153: 396-401.

10 Somppi E, Tammela O, Ruuska $\mathrm{T}$, et al. Outcome of patients operated on for esophageal atresia: 30 years' experience. J Pediatr Surg 1998; 33: 1341-1346.

11 Chetcuti P, Myers NA, Phelan PD, et al. Adults who survived repair of congenital oesophageal atresia and tracheo-oesophageal fistula. BMJ 1988; 297: 344-346.

12 Taylor ACF, Breen KJ, Auldist A, et al. Gastroesophageal reflux and related pathology in adults who were born with esophageal atresia: a long-term follow-up study. Clin Gastroenterol Hepatol 2007; 5: 702-706.

13 Chetcuti P, Phelan PD. Respiratory morbidity after repair of oesophageal atresia and tracheo-oesophageal fistula. Arch Dis Child 1993; 68: 167-170.

14 Agrawal L, Beardsmore CS, MacFadyen UM. Respiratory function in childhood following repair of oesophageal atresia and tracheoesophageal fistula. Arch Dis Child 1999; 81: 404-408.

15 LeSouëf PN, Myers NA, Landau LI. Etiologic factors in long-term respiratory function abnormalities following esophageal atresia repair. J Pediatr Surg 1987; 22: 918-922.

16 Von Hertzen L, Mäkelä MJ, Petäys T, et al. Growing disparities in atopy between the Finns and the Russians: a comparison of 2 generations. J Allergy Clin Immunol 2006; 117: 151-157.

17 Pallasaho $\mathrm{P}$, Lundbäck $\mathrm{B}$, Meren $\mathrm{M}$, et al. Prevalence and risk factors for asthma and chronic bronchitis in the capitals Helsinki, Stockholm, and Tallinn. Respir Med 2002; 96: 759-769.

18 Milligan DWA, Levison $\mathrm{H}$. Lung function in children following repair of tracheoesophageal fistula. J Pediatr 1979; 95: 24-27.

19 Biller JA, Allen JL, Schuster SR, et al. Long-term evaluation of esophageal and pulmonary function in patients with repaired esophageal atresia and tracheoesophageal fistula. Dig Dis Sci 1987; 32: 985-990.

20 Couriel JM, Hibbert M, Olinsky A, et al. Long term pulmonary consequences of oesophageal atresia with tracheo-oesophageal fistula. Acta Paediatr Scand 1982; 71: 973-978.

21 Chetcuti P, Phelan PD, Greenwood R. Lung function abnormalities in repaired oesophageal atresia and tracheo-oesophageal fistula. Thorax 1992; 47: 1030-1034.

22 Sistonen SJ, Koivusalo A, Nieminen U, et al. Esophageal morbidity and function in adults with repaired esophageal atresia with tracheoesophageal fistula: a population-based long-term followup. Annals of Surgery 2010; 251: 1167-1173.

23 Sistonen SJ, Koivusalo A, Lindahl H, et al. Cancer after repair of esophageal atresia: population-based long-term follow-up. J Pediatr Surg 2008; 43: 602-605.

24 Sistonen SJ, Helenius I, Peltonen J, et al. Natural history of spinal anomalies and scoliosis associated with esophageal atresia. Pediatrics 2009; 124: e1198-e1204.

25 Pekkanen J, Remes ST, Husman T, et al. Prevalence of asthma symptoms in video and written questionnaires among children in four regions of Finland. Eur Respir J 1997; 10: 1787-1794.

26 Juniper EF, Guyatt GH, Cox FM, et al. Development and validation of Mini Asthma Quality of Life Questionnaire. Eur Respir J 1999; 14: 32-38.

27 Quanjer PLH, Tammeling GJ, Cotes JE, et al. Lung volumes and forced expiratory flows. Official statement of the European Respiratory Society. Eur J Respir 1993; 6: Suppl. 16, 5-40. 
28 Sovijarvi ARA, Malmberg LP, Reinikainen K, et al. A rapid dosimetric method with controlled tidal breathing for histamine challenge: repeatability and distribution of bronchial reactivity in a clinical material. Chest 1993; 104: 164-170.

29 Viljanen A. Reference values for spirometric, pulmonary diffuse capacity and body plethysmographic studies. Scand J Clinic Laboratory Investig 1982; 42: 5-21.

30 American Thoracic Society. Recommendations for standardized procedures for the online and offline measurement of exhaled lower respiratory nitric oxide and nasal nitric oxide in adults and children - 1999. Am J Respir Crit Care Med 1999; 160: 2104-2117.

31 Ekroos H, Tuominen J, Sovijarvi ARA. Exhaled nitric oxide and its long-term variation in healthy non-smoking subjects. Clin Physiol 2000; 20: 434-439.

32 Taylor DR, Pijnenburg MW, Smith AD, et al. Exhaled nitric oxide measurements: clinical application and interpretation. Occasional Review. Thorax 2006; 61: 817-827.
33 Olin AC. Fraction of exhaled nitric oxide at $50 \mathrm{~mL} \cdot \mathrm{s}^{-1}$. Reference values for adult lifelong never-smokers. Chest 2007; 131: 1852-1856.

34 Pallasaho P, Ronmark E, Haahtela T, et al. Degree and clinical relevance of sensitization to common allergens among adults: a population study in Helsinki, Finland. Clinic Exp Allerg 2006; 36: 503-509.

35 Kotaniemi JT, Pallasaho P, Sovijärvi AR, et al. Respiratory symptoms and asthma in relation to cold climate, inhaled allergens, and irritants: comparison between northern and southern Finland. J Asthma 2002; 39: 649-658.

36 Robertson DF, Mobaireek K, Davis GM, et al. Late pulmonary function following repair of tracheoesophageal fistula or esophageal atresia. Pediatr Pulmonol 1995; 20: 21-26.

37 Juusela M, Poussa T, Kotaniemi J, et al. Bronchial hyperresponsiveness in a population of north Finland with no previous diagnosis of asthma or chronic bronchitis assessed with histamine and methacholine tests. Int J Circumpolar Health 2008; 67: 308-317. 